\title{
A one-dimensional complete hydrodynamic model of border irrigation based on a hybrid numerical method
}

\author{
Shaohui Zhang $\cdot$ Di Xu $\cdot$ Yinong Li
}

Received: 29 October 2009/Accepted: 29 December 2010/Published online: 23 January 2011

(C) Springer-Verlag 2011

\begin{abstract}
A numerical model of border irrigation flow is a useful tool in the design and evaluation of surface irrigation systems. A one-dimensional complete hydrodynamic numerical model of border irrigation was established by using the time-space hybrid numerical method. Differences in stability, convergence, precision, and efficiency of the one-dimensional model were analyzed and compared between the hybrid numerical method proposed here and the Roe finite-volume method. At the same time, the computational performance and simulation effects were validated based on the results of typical border irrigation tests. The results show that the hybrid numerical method provides better numerical stability and convergence with little water quantity-balance and average relative errors than does the Roe finite-volume method. The computational efficiency is about two times higher under the same measurement circumstances. The proposed model of border irrigation can increase computational stability and convergence, can improve computational precision and efficiency, and can provide a good numerical simulation tool for the design and evaluation of border irrigation systems.
\end{abstract}

Communicated by T. Trooien.

S. Zhang $(\bowtie) \cdot$ D. Xu $\cdot$ Y. Li

National Center of Efficient Irrigation Engineering and Technology Research, China Institute of Water Resources and Hydropower Research, Beijing 100048, China

e-mail: zhangsh@iwhr.com

\section{Introduction}

A numerical model of border irrigation is a useful tool in the design and evaluation of surface irrigation systems (Vivekanand and Bhallamudi 1996).

The complete hydrodynamic model, based on the SaintVenant equations, is commonly used to describe the unsteady flow of water in border irrigation. Due to the complexity and large computer execution time required to solve complete hydrodynamic equations, reduced forms of the model, such as the zero-inertia and the kinematic-wave models, have been developed to simulate irrigation flow (Strelkoff and Katopodes 1977; Chen 1970). The zero-inertia model is based on the assumption that the inertia and acceleration terms would be negligible and can accurately simulate flow conditions when the Froude number is very small (Bradford and Katopodes 2001). In the kinematic-wave model, the inertia and free-surface slope terms are neglected; therefore, the model is limited to sloped conditions and cannot handle any downstream boundary conditions that affect upstream flow (Walker and Skogerboe 1987).

Due to recent advances in computer technology, numerical methods such as the finite-difference method and the Roe finite-volume method can be implemented to model surface water flow in border irrigation. However, a numerical vibration exists in the neighborhood of the flow front in the finite-difference model (García-Navarro et al. 2000), and the bottom elevation term cannot be precisely equivalent to other terms (Bradford and Sanders 2005). The Roe finite-volume method can effectively simulate the flow front effect and can depress the vibration (Brufau et al. 2000; Bradford and Katopodes 2001), but it requires substantial computer execution time and lacks practicability due to the constraints of the explicit time scheme and the complex space scheme (LeVeque 2002). 
It is difficult to achieve harmonization in computational precision, efficiency, numerical stability, and convergence. It is thus necessary to use different methods to compute and to maximize each of these different components while shortening the execution time.

Based on the implicit-explicit time scheme, in this study we implemented the finite-difference method, the finitevolume method, and the finite-element method to discretize the different vector terms of the complete hydrodynamic model, and the forming algebraic equation group with coefficient matrix of a three-diagonal shape was solved, and a one-dimensional complete hydrodynamic numerical model of border irrigation was established. The model was validated by using data obtained from typical border irrigation tests.

\section{Establishment of a one-dimensional complete hydrodynamic numerical model of border irrigation based on the hybrid method}

Governing equations of the complete

hydrodynamic model

The unsteady overland flow occurring during one-dimensional border irrigation is generally governed by the continuity and momentum equations, which are generally known as the Saint-Venant equations (Walker and Skogerboe 1987):

$\frac{\partial h}{\partial t}+\frac{\partial q}{\partial x}=-k \alpha \tau^{\alpha-1}$

$\frac{\partial q}{\partial t}+\frac{\partial}{\partial x}(q u)=-\frac{\partial}{\partial x}\left(\frac{g h^{2}}{2}\right)-g h \frac{\partial z}{\partial x}-g \frac{n^{2} u|u|}{h^{1 / 3}} \frac{1}{2} u k \alpha \tau^{\alpha-1}$,

where $h=$ water depth (m); $x=$ the space coordinate $(\mathrm{m})$; $q=$ unit discharge along the coordinate direction $x\left(\mathrm{~m}^{3} /\right.$ (s m) $; \alpha=$ empirical exponent of the empirical Kostiakov equation; $k=$ empirical coefficient of the empirical Kostiakov equation $\left(\mathrm{m} / \mathrm{s}^{\alpha}\right) ; \tau=$ amount of time that any point of the field surface remains wet $(\mathrm{s}) ; t=$ time of border irrigation $(\mathrm{s}) ; g=$ the acceleration due to gravity $\left(\mathrm{m} / \mathrm{s}^{2}\right)$; $u=$ vertically averaged flow velocity $(\mathrm{m} / \mathrm{s}) ; z=$ bottom elevation (m); and $n=$ the Manning roughness coefficient $\left(\mathrm{m}^{1 / 6}\right)$.

By making a transposition in Eq. (2) and then assembling $\frac{\partial}{\partial x}(q u)$ and $\frac{\partial}{\partial x}\left(\frac{g h^{2}}{2}\right)$, the equation becomes:

$\frac{\partial q}{\partial t}+\frac{\partial}{\partial x}\left(q u+\frac{1}{2} g h^{2}\right)=-g h \frac{\partial z}{\partial x}-g \frac{n^{2} u|u|}{h^{1 / 3}} \frac{1}{2} u k \alpha \tau^{\alpha-1}$.
Marking as follows from Eqs. (1) and (3):

$$
\begin{gathered}
\mathbf{U}=\left(\begin{array}{l}
h \\
q
\end{array}\right) ; \quad \mathbf{F}=\left(\begin{array}{c}
q \\
q u+\frac{1}{2} g h^{2}
\end{array}\right) ; \\
\mathbf{S}=\left(\begin{array}{c}
-k \alpha \tau^{\alpha-1} \\
-g h \frac{\partial z}{\partial x}-g \frac{n^{2} u|u|}{h^{1 / 3}} \frac{1}{2} u k \alpha \tau^{\alpha-1}
\end{array}\right),
\end{gathered}
$$

the one-dimensional conserved vector equation of the complete hydrodynamic model is expressed as:

$\frac{\partial \mathbf{U}}{\partial t}+\frac{\partial \mathbf{F}}{\partial x}=\mathbf{S}$

where $\mathbf{U}=$ vector of the conserved dependent variables; $\mathbf{F}=$ the physical flux, containing both the advection physical flux $\mathbf{F}^{(u)}$ and the pressure physical flux $\mathbf{F}^{(p)}$; $\mathbf{S}=$ the source vector containing the bottom elevation vector $\mathbf{S}_{1}$, the bed roughness vector $\mathbf{S}_{2}$, and the infiltration vector $\mathbf{S}_{3}$, which are given as:

$$
\begin{aligned}
\mathbf{F}^{(u)} & =\left(\begin{array}{c}
q \\
q u
\end{array}\right) ; \quad \mathbf{F}^{(p)}=\left(\begin{array}{c}
0 \\
\frac{1}{2} g h^{2}
\end{array}\right) ; \quad \mathbf{S}_{1}=\left(\begin{array}{c}
0 \\
-g h \frac{\partial z}{\partial x}
\end{array}\right) ; \\
\mathbf{S}_{2} & =\left(\begin{array}{c}
0 \\
-g \frac{n^{2} u|u|}{h^{1 / 3}}
\end{array}\right) ; \quad \mathbf{S}_{3}=\left(\begin{array}{c}
-k \alpha^{\alpha-1} \\
\frac{1}{2} u k \alpha \tau^{\alpha-1}
\end{array}\right)
\end{aligned}
$$

The hybrid numerical method

Regarding the time discretization for the governing equations of the proposed model, the Crank-Nicolson implicit time scheme was implemented to $\partial \mathbf{U} / \partial t, \partial \mathbf{F} / \partial x$, and $\mathbf{S}_{2}$ of Eq. (5) at an unknown time $(w+1)$ and a known time $w$, and then $\mathbf{S}_{1}$ and $\mathbf{S}_{3}$ were discretized by using the explicit time scheme at a known time $w$ in order to improve numerical stability and convergence. Concerning the space discretization for the governing equations, based on the reconstructed value of each variable on both faces of the cell boundary (by using the variable value of the cell center to achieve second-order accuracy of each variable), the finite-difference method was firstly employed to discretize the space derivative of the physical flux linear approximation from the implicit time scheme, and an algebraic equation group with coefficient matrix of a three-diagonal shape was formed, which can improve computational efficiency. Secondly, the AUSM finite-volume method with conserved characteristics was used to discretize the space derivative of the advection physical flux and to construct a self-adaptive upwind scheme with non-empirical parameters, and this can eliminate any spurious oscillations, then the central scheme finite-volume method with conserved characteristics was used to discretize the space derivative of the pressure physical flux in order to improve computational efficiency and weaken numerical dissipation. Finally, the finite-element method with 
conserved characteristics was implemented to discretize the bottom element vector with the non-space derivative term $h$, and then the bed roughness vector and the infiltration vector were, respectively calculated according to the corresponding space-node values. The Thomas method was used to solve time-space discretized algebraic equations with coefficient matrix of a three-diagonal shape.

Time discretization of the governing equation of the one-dimensional complete hydrodynamic model of border irrigation

The Crank-Nicolson implicit time scheme was employed to $\partial \mathbf{U} / \partial t, \partial \mathbf{F} / \partial x$, and $\mathbf{S}_{2}$, and the explicit time scheme was applied to $\mathbf{S}_{1}$ and $\mathbf{S}_{3}$, and then the implicit-explicit hybrid time scheme of Eq. (5) can be given as:

$$
\begin{gathered}
\frac{\mathbf{U}^{w+1}-\mathbf{U}^{w}}{\Delta t}+\frac{1}{2}\left[\left(\frac{\partial \mathbf{F}}{\partial x}\right)^{w+1}+\left(\frac{\partial \mathbf{F}}{\partial x}\right)^{w}\right] \\
=\mathbf{S}_{1}^{w}+\frac{1}{2}\left(\mathbf{S}_{2}^{w+1}+\mathbf{S}_{2}^{w}\right)+\mathbf{S}_{3}^{w},
\end{gathered}
$$

where $\Delta t=$ the time step size, $(\mathrm{t})$.

Expanding the physical flux $\mathbf{F}$ of Eq. (7) by using the Tailor formula and retaining the linear terms:

$\mathbf{F}^{w+1}=\mathbf{F}^{w}+\left(\frac{\partial \mathbf{F}}{\partial \mathbf{U}}\right)^{w}\left(\mathbf{U}^{w+1}-\mathbf{U}^{w}\right)=\mathbf{F}^{w}+\mathbf{A}^{w} \Delta \mathbf{U}^{w}$,

where $\mathbf{A}$ is the Jacobin matrix.

By making a linear approximation of $\mathbf{S}_{2}^{\omega+1}$ in Eq. (7):

$$
\mathbf{S}_{2}^{w+1}=\mathbf{f}^{w} \cdot \mathbf{U}^{w+1}=\left(\begin{array}{cc}
0 & 0 \\
0 & \frac{g n^{2}|u|}{h^{4 / 3}}
\end{array}\right)^{w}\left(\begin{array}{l}
h \\
q
\end{array}\right)^{w+1} .
$$

By substituting Eqs. (8) and (9) into Eq. (7) and marking $\Delta \mathbf{U}^{w}=\mathbf{U}^{w+1}-\mathbf{U}^{\mathrm{w}}$, the delta type of the implicit-explicit hybrid time scheme of Eq. (5), can be expressed as:

$$
\begin{aligned}
(\mathbf{I} & \left.+\mathbf{f}^{w}\right) \Delta \mathbf{U}^{w}+\frac{\Delta t \partial}{2 \partial x}(\mathbf{A} \cdot \Delta \mathbf{U})^{w} \\
& =-\Delta t\left(\frac{\partial \mathbf{F}}{\partial x}\right)^{w}+\Delta t\left[\left(\mathbf{S}_{1}\right)^{w}+\left(\mathbf{S}_{2}\right)^{w}+\left(\mathbf{S}_{3}\right)^{w}\right],
\end{aligned}
$$

where $\mathbf{I}$ is the unit matrix.

Space discretization of the governing equation of the one-dimensional complete hydrodynamic model of border irrigation

Reconstructed values of the physical variables Water depth $\left(h_{i}+1 / 2\right)_{L}$ and $\left(h_{i}+1 / 2\right)_{R}$ on both faces of each cell $i$ boundary $(i+1 / 2)$ was reconstructed by using the water depth value of the cell center as:

$\left(h_{i+1 / 2}\right)_{L}=h_{i}+\frac{1}{2} \Delta h_{i}$ $\left(h_{i+1 / 2}\right)_{R}=h_{i}-\frac{1}{2} \Delta h_{i+1}$.

The $\Delta h_{i}$ and $\Delta h_{i+1}$ in Eqs. (11) and (12) were calculated by using the min mod flux-limiter in order to eliminate spurious oscillations and to make the water depth values reach second-order accuracy along the computational field (LeVeque 2002) as such:

$\Delta h_{i}=\min \bmod \left(h_{i+1}-h_{i}, h_{i}-h_{i-1}\right)$

$\Delta h_{i+1}=\min \bmod \left(h_{i+1}-h_{i}, h_{i+2}-h_{i+1}\right)$.

The process of reconstructed values of the other physical variables, such as flow velocity $u$ and unit discharge $q$, on both faces of each cell $i$ boundary $(i+1 / 2)$ was the same as for the water depth $h$.

Finite-difference method discretized scheme of the space derivative of the physical flux linear approximation $\frac{\partial}{\partial x}(\mathbf{A} \cdot \Delta \mathbf{U})^{w} \quad$ The space derivative of the physical flux linear approximation $\frac{\partial}{\partial x}(\mathbf{A} \cdot \Delta \mathbf{U})^{w}$ in Eq. (10) was space discretized by using the central-difference scheme of the finite-difference method at cell $i$ in terms of the values at the cell boundaries, $i-1 / 2$ and $i+1 / 2$, and $\mathbf{f}^{w}$ is calculated by using cell $i$ center values:

$$
\begin{aligned}
(\mathbf{I} & \left.+\mathbf{f}_{i}^{w}\right) \Delta \mathbf{U}_{i}^{w}+\frac{\Delta t}{x_{i+1}-x_{i-1}}\left[(\mathbf{A} \cdot \Delta \mathbf{U})_{i+1}^{w}-(\mathbf{A} \cdot \Delta \mathbf{U})_{i-1}^{w}\right] \\
& =-\Delta t\left(\frac{\partial \mathbf{F}}{\partial x}\right)^{w}+\Delta t\left[\left(\mathbf{S}_{1}\right)^{w}+\left(\mathbf{S}_{2}\right)^{w}+\left(\mathbf{S}_{3}\right)^{w}\right]
\end{aligned}
$$

where $x_{i-1}$ and $x_{i+1}$ are, respectively, the center coordinates of the spatial cell $(i-1)$ and $(i+1)$.

After a rearrangement, Eq. (15) can be expressed as:

$$
\begin{aligned}
& -\frac{\Delta t}{x_{i+1}-x_{i-1}}(\mathbf{A} \cdot \Delta \mathbf{U})_{i-1}^{w}+\left(\mathbf{I}+\mathbf{f}_{i}^{w}\right) \Delta \mathbf{U}_{i}^{w}+\frac{\Delta t}{x_{i+1}-x_{i-1}} \\
& \times(\mathbf{A} \cdot \Delta \mathbf{U})_{i+1}^{w}=-\Delta t\left(\frac{\partial \mathbf{F}}{\partial x}\right)^{w}+\Delta t\left[\left(\mathbf{S}_{1}\right)^{w}+\left(\mathbf{S}_{2}\right)^{w}+\left(\mathbf{S}_{3}\right)^{w}\right] .
\end{aligned}
$$

Finite-volume method discretized scheme of the space derivative of the physical flux $\left(\frac{\partial \mathbf{F}}{\partial x}\right)^{w}$ The finite-volume method was employed to discretize the space derivative of the physical flux $\left(\frac{\partial \mathbf{F}}{\partial x}\right)^{w}$, which was area averaged on each cell $i$ and then the divergence theorem was applied:

$\frac{1}{\Delta x_{i}} \int_{x_{i-1 / 2}}^{x_{i+1 / 2}}\left(\frac{\partial \mathbf{F}}{\partial x}\right)^{w} \mathrm{~d} x=\frac{1}{\Delta x_{i}}\left(\mathbf{F}_{i+1 / 2}^{*}-\mathbf{F}_{i-1 / 2}^{*}\right)$,

where $\mathbf{F}_{i+1 / 2}^{*}$ and $\mathbf{F}_{i-1 / 2}^{*}$ are the numerical fluxes, and $x_{i-1 / 2}$ and $x_{i+1 / 2}$ are the left and right boundary coordinates, respectively, of cell $i$. 
$\mathbf{F}_{i+1 / 2}^{*}$ in Eq. (17) is the sum of the advection and pressure terms: $\mathbf{F}_{i+1 / 2}^{*}=\mathbf{F}_{i+1 / 2}^{(u) *}+\mathbf{F}_{i+1 / 2}^{(p) *}, \mathbf{F}_{i+1 / 2}^{(u) *}$ was discretized by using the AUSM scheme (Liou 1996), and the expression of $\mathbf{F}_{i+1 / 2}^{(p) *}$ by using the central scheme is written as (LeVeque 2002):

$\mathbf{F}_{i+1 / 2}^{(p)}=\frac{1}{2}\left(\begin{array}{c}0 \\ g h_{i+1 / 2}^{2} / 2\end{array}\right)_{L}+\frac{1}{2}\left(g h_{i+1 / 2}^{2} / 2\right)_{R}$.

The discretization process for numerical flux $\mathbf{F}_{i-1 / 2}^{*}$ in Eq. (17) was the same as for $\mathbf{F}_{i+1 / 2}^{*}$.

Finite-element method discretized scheme of bottom elevation $\left(\mathbf{S}_{1}\right)^{w} \quad$ By selecting the weighting function of each cell as 1 , the bottom elevation vector $\left(\mathbf{S}_{1}\right)^{w}$ of Eq. (10) was integrated over the interval $\left[x_{i}-1 / 2, x_{i}+1 / 2\right]$ corresponding to cell $i$, and then the integral was area averaged to obtain $\left(\mathbf{S}_{1}^{*}\right)_{i}^{w}$ :

$$
\begin{gathered}
\left(\mathbf{S}_{1}^{*}\right)_{i}^{w}=\frac{1}{x_{i+1 / 2}-x_{i-1 / 2}} \int_{x_{i-1 / 2}}^{x_{i+1 / 2}} 0 \\
\left(\mathbf{S}_{1}\right)^{w} \mathrm{~d} x=\left(\begin{array}{c}
0 \\
\frac{1}{x_{i+1 / 2}-x_{i-1 / 2}} \int_{x_{i-1 / 2}}^{x_{i+1 / 2}} g h \frac{\partial z}{\partial x} d x
\end{array}\right) .
\end{gathered}
$$

The water depth $h$ and the bottom elevation $z$ in Eq. (19) were linear approximated by using the linear Langrange function:

$$
\begin{gathered}
h=\left(h_{i-1 / 2}\right)_{R} \frac{x_{i+1 / 2}-x}{x_{i+1 / 2}-x_{i-1 / 2}}+\left(h_{i+1 / 2}\right)_{L} \frac{x-x_{i-1 / 2}}{x_{i+1 / 2}-x_{i-1 / 2}}, \\
z=z_{i-1 / 2} \frac{x_{i+1 / 2}-x}{x_{i+1 / 2}-x_{i-1 / 2}}+z_{i+1 / 2} \frac{x-x_{i-1 / 2}}{x_{i+1 / 2}-x_{i-1 / 2}}
\end{gathered}
$$

where $z_{i-1 / 2}$ and $z_{i+1 / 2}$ are the bottom elevation values on side $(i-1 / 2)$ and $(i+1 / 2)$ of cell $i$, respectively $(\mathrm{m})$; and the meaning of other symbols are as described above.

Substituting Eq. (20) into Eq. (19), and the space discretized scheme of $\left(\mathbf{S}_{1}^{*}\right)_{i}^{w}$ becomes:

$\left(\mathbf{S}_{1}^{*}\right)_{i}^{w}=\left(g \frac{\left(z_{i+1 / 2}-z_{i-1 / 2}\right)\left[\left(h_{i-1 / 2}\right)_{R}+\left(h_{i+1 / 2}\right)_{L}\right]}{2\left(x_{i+1 / 2}-x_{i-1 / 2}\right)}\right)$.

The roughness vector $\mathbf{S}_{2}$ and the infiltration vector $\mathbf{S}_{3}$ are non-derivative terms and do not need space discretization; they are calculated directly according to the center values of cell $i$.

\section{Solution of the time-space discretized scheme of the governing equation}

As above, after time-space discretization of the governing equation of the one-dimensional complete hydrodynamic model, the final numerical discretized scheme of Eq. (5) is expressed as:

$$
\begin{gathered}
-\frac{\Delta t}{2 \Delta x_{i}}\left[(\mathbf{A} \cdot \Delta \mathbf{U})_{i-1 / 2}^{w}\right]_{R}+\left(\mathbf{I}+\mathbf{f}_{i}^{w}\right) \Delta \mathbf{U}_{i}^{w}+\frac{\Delta t}{2 \Delta x_{i}}\left[(\mathbf{A} \cdot \Delta \mathbf{U})_{i+1 / 2}^{w}\right]_{L} \\
=-\frac{\Delta t}{\Delta x_{i}}\left(\mathbf{F}_{i+1 / 2}^{*}-\mathbf{F}_{i-1 / 2}^{*}\right)^{w}+\Delta t\left[\left(\mathbf{S}_{1}^{*}\right)_{i}^{w}+\left(\mathbf{S}_{2}\right)_{i}^{w}+\left(\mathbf{S}_{3}\right)_{i}^{w}\right]
\end{gathered}
$$

Equation (22) is a linear algebraic equation group with coefficient matrix of a three-diagonal shape, and the dependent variable vector $\mathbf{U}$ contains two components. Thus, the coefficient matrix element of Eq. (5) is a matrix. Equation (22) was solved by using the Thomas method, and then the value of $\mathbf{U}_{i}^{w+1}$ was obtained according to the formula $\mathbf{U}_{i}^{w+1}=\mathbf{U}_{i}^{w}+\Delta \mathbf{U}_{i}^{w}$ (Hildebrand 1974).

Initial and boundary conditions and stability

\section{Initial conditions}

When $t=0$, the water depth in each cell was zero, and every point in the computational field was the singularity of the discretized governing equations. In order to begin the computation, the initial values of $h$ should be obtained according to the numerical tests. The criteria for selecting the initial value are that the numerical results should be stable and the initial values should not have a significant effect on the numerical results; that is, errors between different initial values are smaller than the numerical values by two orders of magnitude, and the recommended initial value is $h_{\text {initial }}=10^{-10} \mathrm{~m}$. In the advance and recession phases of border irrigation, a boundary exists between the dry and wet bed. The simulations do not directly track this boundary, but judge the water depth value at every cell center by using the definition of initial water depth $h_{\text {initial }}$. When the water depth of the cell center is below $h_{\text {initial }}$, the water depth $h$ should be equal to $h_{\text {initial }}$ and the cell is dry; otherwise it is wet. The initial values of $q$ and $u$ in each cell are equal to zero.

\section{Boundary conditions of upstream and downstream}

When the upstream portion is in the inflow condition, for the finite-volume and finite-element methods, the boundary cell center of upstream is the giving unit discharge $q=q_{0}$, and the corresponding water depth $h$ is:

$h=\max \left(h_{\mathrm{c}}, h_{\text {critical }}\right)$,

where $h_{\mathrm{c}}$ is the water depth in the cell center of upstream and $h_{\text {critical }}$ is the critical water depth, $h_{\text {critical }}=1.05 \times\left(q^{2}\right)$ $g)^{1 / 3}$ (Brufau et al. 2000).

When the upstream portion is in the inflow condition, for the finite-difference method an additional cell should be 
set close to the outside boundary of the first cell in the upstream, and its center is the giving unit discharge $q=q_{0}$, and the corresponding water depth $h$ should be interpolated from the physical variables of the inner cell.

When the upstream inflow is stopped, for the finitevolume and finite-element methods the discharge and velocity of the first cell center in the upstream is zero, $q=0, u=0$, and the same conditions are set to the additional cell close to the outside boundary of the first cell in the upstream in terms of the finite-difference method.

When the flow of downstream is in the closed condition, for the finite-volume and finite-element methods, the discharge and velocity of the tail cell center in the downstream is zero, $q=0, u=0$, and the same conditions are set to the additional cell close to the outside boundary of the tail cell in the downstream in terms of the finite-difference method. If the downstream flow condition is open, then the discharge and water depth of the tail cell center in the downstream for the finite-volume and the finite-element methods should be interpolated from the inner cell values. This is done for the additional cell close to the outside boundary of the tail cell in the upstream in terms of the finite-difference method.

\section{Bottom conditions}

Corresponding to the space scheme of the second-order accuracy of the discretized governing equations, the bottom elevation data of each computational point in the border was interpolated by using the non-uniform rational B-spline curves function based on the measured data (Pieg 1989).

\section{Stability}

The stability condition of Eq. (22) depends on choosing appropriate values of $\Delta t / \Delta x$. When $\Delta t / \Delta x$ is within some value range, the results are stable. On the contrary, errors from simulation would accumulate to make the computational results unstable. Owing to the strongly nonlinear characteristics of Eq. (22), the strict stability analysis theory cannot provide the sufficient conditions. Consequently, the stability of Eq. (22) would be confirmed by the stability of the computational results during the numerical simulations.

\section{Evaluation and validation of the proposed model}

Test and method

\section{Typical border irrigation test examples}

Four typical border irrigation tests were chosen as examples. Each test was different in several technical elements, such as border length, unit discharge, surface slope, and bottom elevation standard deviation. Differences in stability, convergence, precision, and efficiency were analyzed, and the computational performance and simulation effects were validated between the proposed numerical method and the Roe finite-volume method. Data for example 1 in Table 1 are from winter wheat irrigation tests in Xiong County, Hebei Province, China. The surface soil was a silty loam, and the dry bulk density was $1.38 \mathrm{~g} / \mathrm{cm}^{3}$. Data for examples 2, 3, and 4 in Table 1 are from winter wheat irrigation tests in the Bojili irrigation district in Shandong Province, China. The surface soil was a silty loam with a dry bulk density of $1.34 \mathrm{~g} / \mathrm{cm}^{3}$ (Xu et al. 2004). Observational tests of soil infiltration were carried out at the time of the observation of the water depth, and a basin infiltrometer method was used to determine $K$ and $\alpha$ of the Kostiakov empirical infiltration formula, $Z=k \tau^{\alpha}$. The water depth was used to estimate the roughness coefficient $n$ (Xu et al. 2000), and the space between two adjacent measured points of bottom elevation was $10 \mathrm{~m}$ for all examples.

\section{Roe finite-volume method}

In the course of solving the one-dimensional complete hydrodynamic border irrigation model by using Roe finitevolume method, the time derivative $\partial \mathbf{U} / \partial t$ of the dependent variable vector $\mathbf{U}$ was discretized by using the two-stage Runge-Kutta explicit time scheme. The Roe finite-volume method was implemented to discretize the space derivative

Table 1 Observational data of typical border irrigation tests and the measured results of soil parameters

\begin{tabular}{|c|c|c|c|c|c|c|c|c|}
\hline \multirow[t]{2}{*}{$\begin{array}{l}\text { Border irrigation } \\
\text { samples }\end{array}$} & \multirow[t]{2}{*}{$\begin{array}{l}\text { Border } \\
\text { long } L / \mathrm{m}\end{array}$} & \multirow[t]{2}{*}{$\begin{array}{l}\text { Unite discharge } \\
q / \mathrm{L} \mathrm{s}^{-1} \mathrm{~m}^{-1}\end{array}$} & \multirow[t]{2}{*}{$\begin{array}{l}\text { Slope } \\
i / \% \text { o }\end{array}$} & \multirow{2}{*}{$\begin{array}{l}\text { Bottom elevation } \\
\text { standard deviation } \\
S_{\mathrm{d}} / \mathrm{cm}\end{array}$} & \multirow[t]{2}{*}{$\begin{array}{l}\text { Irrigation } \\
\text { time } t / \mathrm{min}\end{array}$} & \multicolumn{2}{|l|}{$\begin{array}{l}\text { Infiltration } \\
\text { parameter }\end{array}$} & \multirow[t]{2}{*}{$\begin{array}{l}\text { Roughness } \\
\text { coefficient }\end{array}$} \\
\hline & & & & & & $k / \mathrm{mm} \mathrm{h}^{-\alpha}$ & $\alpha$ & \\
\hline 1 & 55 & 3.79 & 1.30 & 2.40 & 56 & 160 & 0.50 & 0.20 \\
\hline 2 & 80 & 1.78 & 0.40 & 0.82 & 74 & 75 & 0.60 & 0.10 \\
\hline 3 & 140 & 2.71 & 1.23 & 5.81 & 146 & 92 & 0.53 & 0.14 \\
\hline 4 & 280 & 4.66 & 1.30 & 3.06 & 185 & 110 & 0.45 & 0.13 \\
\hline
\end{tabular}


$\partial \mathbf{F} / \partial x$ of the physical flux $\mathbf{F}$ and the bottom elevation $\mathbf{S}_{1}$ of the source vector $\mathbf{S}$ based on the reconstructed value of each variable on both faces of the cell boundary, and then the time-space discretized formulation was solved (Brufau et al. 2000; LeVeque 2002). In contrast, the hybrid numerical method uses the implicit-explicit hybrid time discretization scheme to form time stepping, and then the finite-difference, the finite-volume, and the finite-element methods are, respectively, implemented to discretize the space derivative of the physical flux linear approximation from the implicit time scheme, the space derivative of the physical flux, and the elevation vector of the source term. It can be seen that the main differences between the above two numerical methods are the solving of the space derivative of the physical flux linear approximation and of the bottom elevation vector. Although both methods are involved in the finite-volume method, the Roe finite-volume method has a complex construction in the space scheme, and the AUSM and central scheme finite-volume methods not only have high precision but also have relatively simpler constructions in the space scheme. In addition, the hybrid numerical method contains the physical flux linear approximation, which is obtained from the implicit time scheme and can improve numerical stability and convergence. The Roe finite-volume method does not provide these advantages.

\section{Results and discussion}

Based on the simulation results of surface flow advance and recession and flow depth obtained by using the proposed method and the Roe finite-volume method, the parameters measuring numerical stability and convergence as well as precision were constructed. The simulation results, such as surface flow advance and recession processes, flow depth process, and water volume balance error varied with respect to the space step size $\Delta x$ (the length of space cell) and the ratio of the time-space step size, $\Delta t / \Delta x$. Thus, the parameters for measuring numerical stability and convergence as well as precision are a function of $\Delta x$ and $\Delta t / \Delta x$. In the twodimensional plan that employs $\Delta x$ and $\Delta t / \Delta x$, supposing that the parameters vary monotonously with respect to the combined area of $\Delta x$ and $\Delta t / \Delta x$, and for any giving parameter values, $\Delta x$ and $\Delta t / \Delta x$ have a larger combined domain based on one numerical method versus another, this means that the parameters have little influence on this numerical method than they do on the other; that is, this numerical method has better computational performance.

\section{Numerical stability}

Measured parameters of numerical stability Numerical stability is the influence of the initial error, the boundary error, and the stepping error on the simulation results is limited (LeVeque 2002). We can introduce the oscillation amplitude of the simulation flow depth $\Delta h$ as parameter for measuring numerical stability, when unit discharge is close to zero $\left(q \leq 0.001 \mathrm{~m}^{3} / \mathrm{s}\right)$ in the recession phase, and usually $\Delta h=0.01 \mathrm{~m}$ (Strelkoff et al. 2003).

When there is one connecting domain in the border, $\Delta h$ is constructed as:

$\Delta h=\max \left[\max \left(h^{w}+z\right)-\min \left(h^{w}+z\right)\right]$

where $h^{w}$ is the simulated values of the water depth along the border at time $w$ and $z$ is the bottom elevation corresponding to $h^{w}$.

When there are many connecting domains in the border $\Delta h$ is expressed as:

$\Delta h=\max \left(\Delta h_{i}^{w}\right)(i=1,2,3, \ldots, N)$,

where $\Delta h_{i}^{w}$ is the oscillation amplitude of the simulation flow depth in connected domains $i$ at time $w$, and $N$ is the number of water-connected domains in the border.

Difference analysis of numerical stability Figure 1 shows the area of the $\Delta x$ versus $\Delta t / \Delta x$ combination domain corresponding to both numerical methods for different waterdepth simulation oscillation amplitude values of $\Delta h=0.01$, 0.005 , and $0.001 \mathrm{~m}$. For all typical border irrigation examples, the area of the proposed hybrid numerical method is larger than it is for the Roe finite-volume method under the different constraints of the measured parameter $\Delta h$. This means that the proposed has better numerical stability than does the Roe finite-volume method. As seen in Fig. 1, example 2 (Fig. 1b) has a relatively larger area than does example 3 (Fig. 1c). This is closely related to the case shown in Table 1, where the former has a relatively flatter bottom $\left(S_{d}=0.82 \mathrm{~cm}\right)$ and the latter has a bottom with relatively large standard derivation value $\left(S_{d}=5.81 \mathrm{~cm}\right)$. Larger values of $S_{d}$ would have an impact on the instantaneously dynamic equilibrium between the bottom elevation vector and other physical vectors and then have an adverse effect on numerical stability.

\section{Numerical convergence}

Measured parameters of numerical convergence Numerical convergence is when the time and space step size $\Delta t$ versus $\Delta x$ tend toward zero, the errors between the numerical results and the analytical solution tend toward infinitesimally small (LeVeque 2002). When an analytical solution does not exist, the time function series from the advance and the recession phases of the surface flow are as follow:

$t(x)_{\mathrm{adv}}^{0}, t(x)_{\mathrm{adv}}^{1} \cdots t(x)_{\mathrm{adv}}^{k_{\mathrm{t}}-1}, t(x)_{\mathrm{adv}}^{k_{\mathrm{t}}}, t(x)_{\mathrm{adv}}^{k_{\mathrm{t}}+1} \cdots$

$t(x)_{\mathrm{rec}}^{0}, t(x)_{\mathrm{rec}}^{1} \cdots t(x)_{\mathrm{rec}}^{k_{\mathrm{t}}-1}, t(x)_{\mathrm{rec}}^{k_{\mathrm{t}}}, t(x)_{\mathrm{rec}}^{k_{\mathrm{t}}+1} \cdots$ 


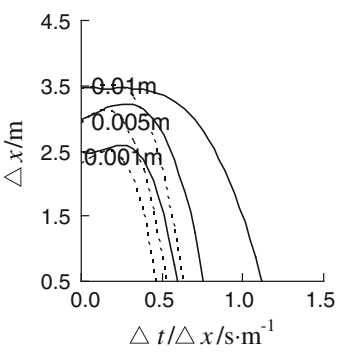

(a) Example 1

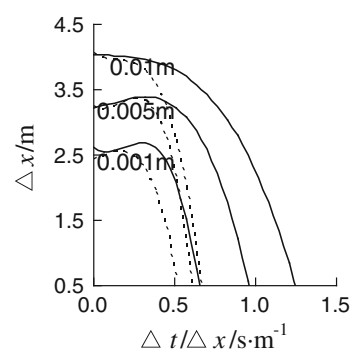

(b) Example 2

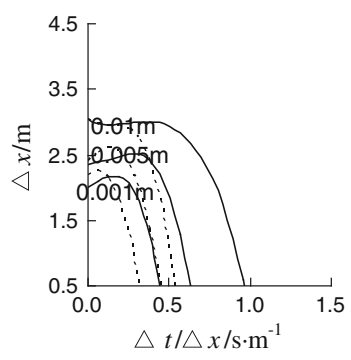

(c) Example 3

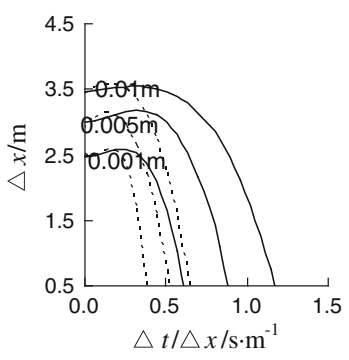

(d) Example 4

— Hybrid numerical method - - - - Roe finite-volume method

Fig. 1 Numerical stability comparison of the two methods for different values of $\Delta \mathrm{h}$. Solid line Hybrid numerical method, dotted line Roe finitevolume method
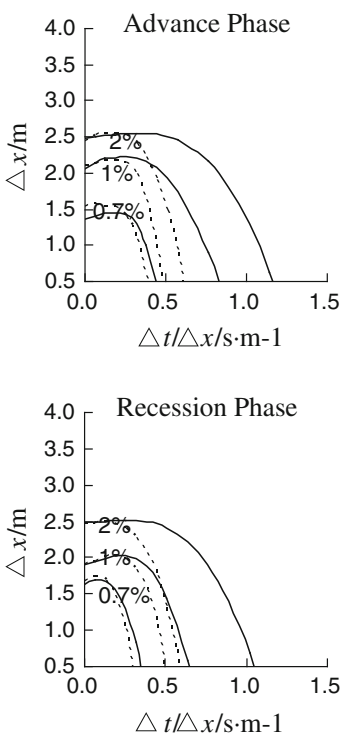

(a) Example 1
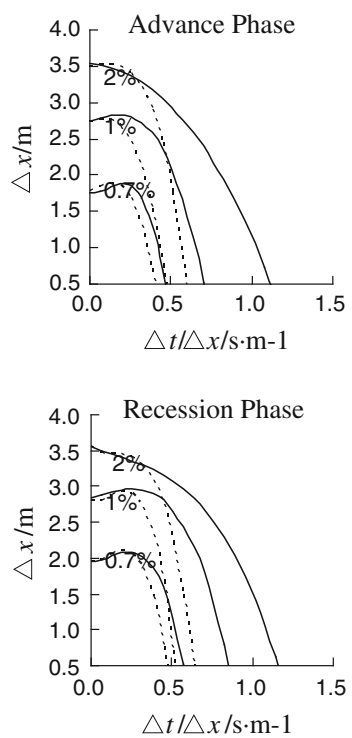

(b) Example 2
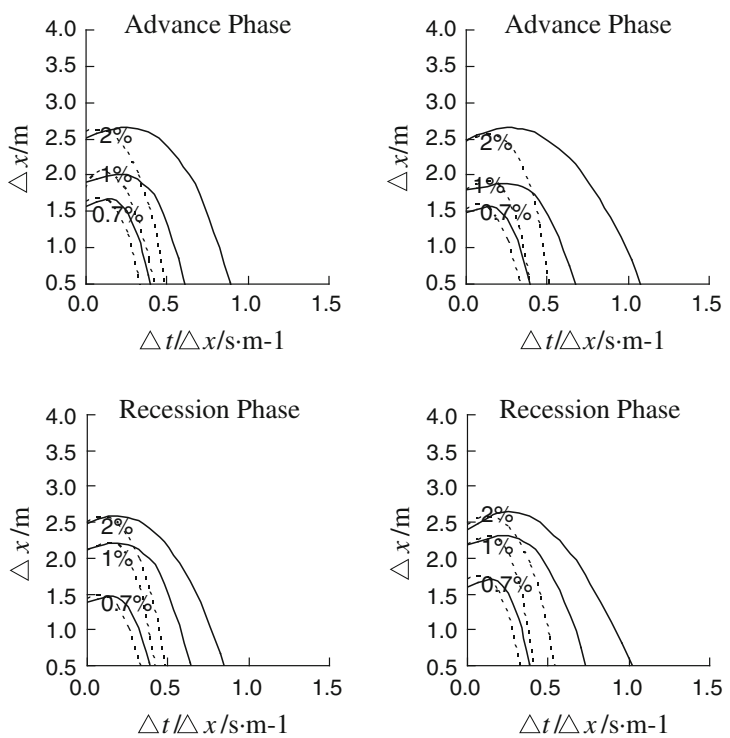

(c) Example 3

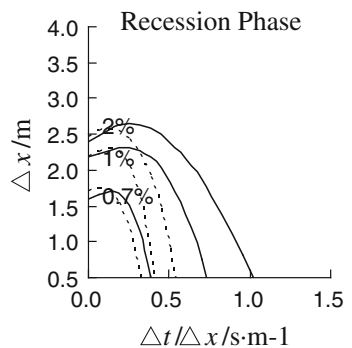

(d) Example 4

Hybrid numerical method -- - Roe finite-volume method

Fig. 2 Numerical convergence comparison of the two numerical methods for different values of $A R E_{k_{t}, k_{t}+1}$. Solid line Hybrid numerical method, dotted line Roe finite-volume method

The average relative error $A R E_{k_{t}, k_{t+1}}$ of two adjacent time functions in series (26) and (27) is used as a parameter to measure numerical convergence, and $A R E_{k_{t}, k_{t+1}}$ is usually equal to $2 \%$ (Lei et al. 1988) but is calculated by:

$A R E_{k_{t}, k_{t}+1}=\frac{1}{M} \sum_{i=1}^{M} \frac{\left|t\left(x_{i}\right)^{k_{t}+1}-t\left(x_{i}\right)^{k_{t}}\right|}{t\left(x_{i}\right)^{k_{t}}} \times 100 \%$,

where $x_{i}=$ the point coordinate of the space cell $i$ center (m); $M=$ the number of space cells; $k_{\mathrm{t}}$ and $k_{\mathrm{t}}+1$ are the superscripts of the two adjacent time function series of flow advance and recession corresponding to $\Delta t$ and $\Delta x$.

Difference analysis of numerical convergence Figure 2 shows the area of the $\Delta x$ versus $\Delta t / \Delta x$ composition domain corresponding to both numerical methods in the case of different $A R E_{k_{t}, k_{t}+1}$ values $(2,1$, and $0.7 \%)$. For all typical border irrigation examples, the area in the flow advance and recession phases is larger than it is in the Roe finitevolume method under the different constraints of the measured parameterARE $E_{k_{t}, k_{t}+1}$. This means that the hybrid numerical method has better numerical convergence than does the Roe finite-volume method. As seen in Fig. 2, the area is larger in example 2 (Fig. 2b) than it is in example 3 (Fig. 2c) in both the advance and recession phases. This shows that a relatively poor bottom elevation would have an adverse influence on numerical convergence.

\section{Computational precision and efficiency}

Measurement parameters of computational precision and efficiency Computational precision can be, in practicality 
of border irrigation, that the water quantity-balance error of the simulation and the average relative error (ARE) between the simulation results and the observational values when satisfying the conditions of numerical stability and convergence. The corresponding measurement parameters include those involved in the water quantity-balance error at the end of irrigation, $A R E_{\mathrm{adv}}$ and $A R E_{\mathrm{rec}}$ of the flow advance and recession times between the simulation results and observational values, respectively (Li 1999), and the water quantity-balance error $e$ as following,

$e=\frac{V_{\text {in }}-\left(V_{\text {surface }}+V_{\text {subsurface }}\right)}{V_{\text {in }}} \times 100 \%$,

where $V_{\text {in }}=$ the quantity of water flowing into the border from an upstream inlet; $V_{\text {surface }}=$ the quantity of water stored in the border; and $V_{\text {subsurface }}=$ the quantity of water infiltrated into the soil.

Computational efficiency is the simulating workload completed per unit of time, and its measurement parameter is $1 / T$, where $T$ is the computer execution time required for one numerical method to finish simulating a specific example.

Difference analysis of water balance error Figure 3 shows the area of the $\Delta x$ versus $\Delta t / \Delta x$ combination domain corresponding to the two numerical methods for different values of water balance error $e$. The area corresponding to the hybrid numerical methods under larger values of $e$ is clearly larger than it is by using the Roe finite-volume method. There is little difference in area for the two numerical methods, when $e$ is small. Overall, the water balance error of the hybrid numerical method was lower than it was when the Roe finite-volume method was used.

Difference analysis of the average relative error Figure 4 shows the area of the $\Delta x$ versus $\Delta t / \Delta x$ composition domain corresponding to the two numerical methods in the case of different values of $A R E_{\mathrm{adv}}$ and $A R E_{\mathrm{rec}}$. For all typical

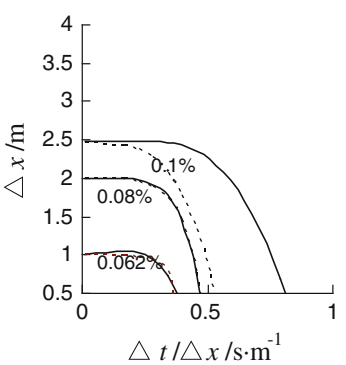

(a) Example 1

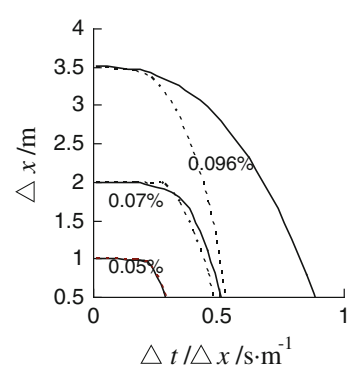

(b) Example 2 border irrigation examples, the area for the proposed hybrid numerical method in the flow advance and recession phases was larger than that found by use of the Roe finite-volume method. This means that the hybrid numerical method has little average relative error between the simulation results and the observational data than does the Roe finite-volume method. On the other hand, the area is larger in example 2 (Fig. 4b) than it is in example 3 (Fig. 4c) for both the flow advance and recession phases. This shows that a relatively poor bottom elevation would have adverse effects on numerical precision.

In order to intuitively present computational precision of hybrid numerical method, in Fig. 4, at most lateral contour of example 3, which has most small area of $\Delta x$ versus $\Delta t$ / $\Delta x, \Delta x=2$ and $\Delta t / \Delta x=0.6$ were selected as time-space step for two numerical methods. Figure 5 shows the comparison of simulated and observed values by numerical methods under different typical border irrigation examples. Fitting degree between observed and simulated values by hybrid numerical method was better. The simulated values of the Roe finite-volume method presented some unstable results, and did not exist at border tail in recession phase for example 1, 3, and 4. That is, effective area of border space field for the Roe finite-volume method was intuitively decreased.

In the effective area of Roe finite-volume method, Table 2 shows average relative errors between simulated and observational values by two numerical methods under typical border irrigation examples corresponding to Fig. 5. As seen in Table 2, even in the effective area of Roe finitevolume method, hybrid numerical method has yet better computational precision.

\section{Difference analysis of computational efficiency}

As seen in Fig. 3, both of the numerical methods have very similar values of water quantity-balance error, when $\Delta x=2 \mathrm{~m}$ and $\Delta t / \Delta x=0.1 \mathrm{~s} / \mathrm{m}$. Thus, based on the values

— Hybrid numerical method - - --Roe finite-volume method

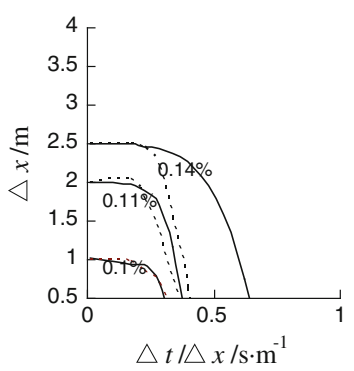

(c) Example 3

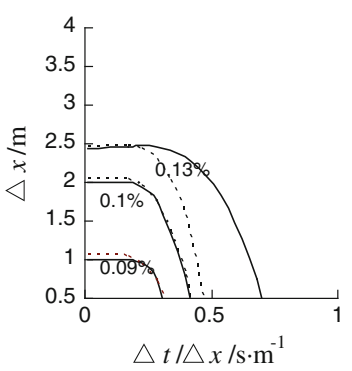

(d) Example 4

Fig. 3 Numerical precision comparison of two numerical methods for different values $e$. Solid line Hybrid numerical method, dotted line Roe finite-volume method 

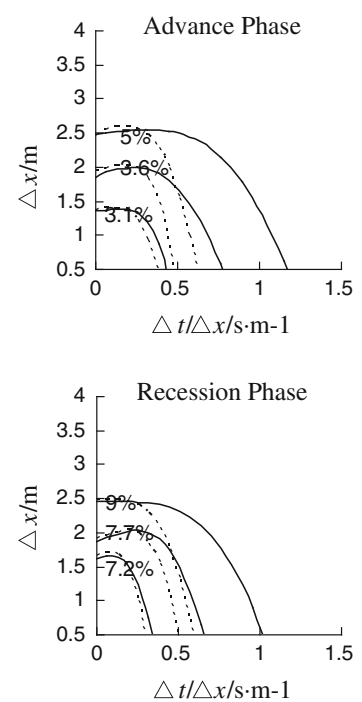

(a) Example 1
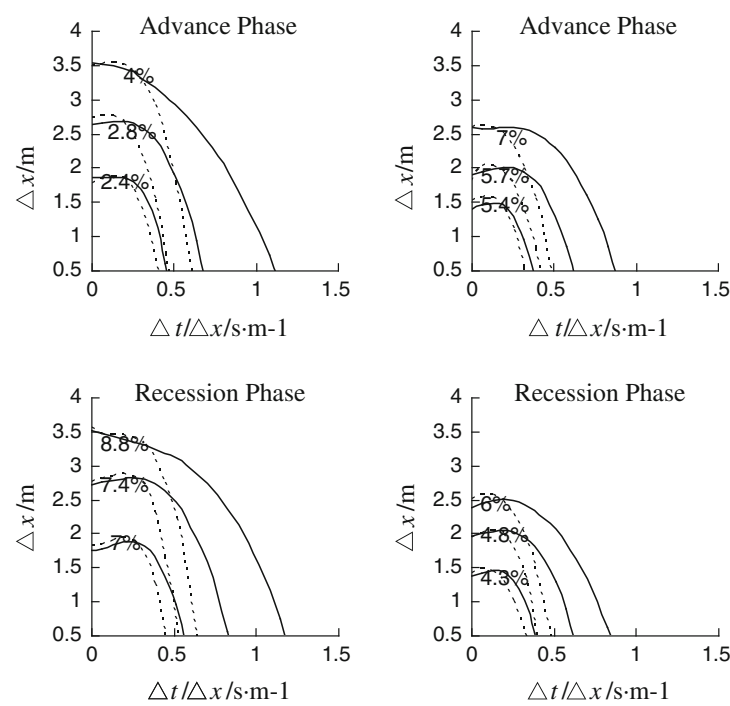

(b) Example 2

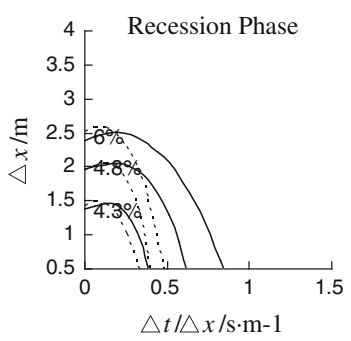

(c) Example 3
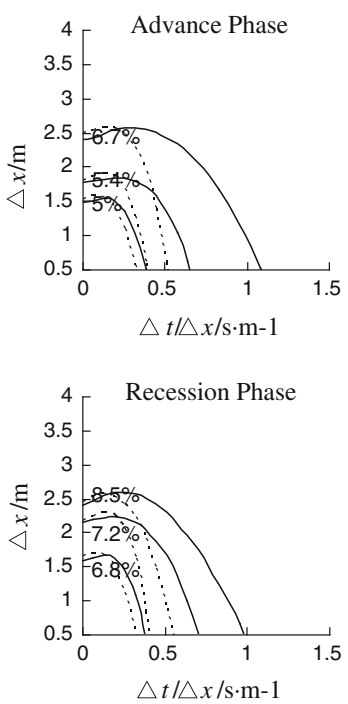

(d) Example 4

— Hybrid numerical method - - - - Roe finite-volume method

Fig. 4 Numerical precision comparison of the two numerical methods for different values of $A R E_{\mathrm{adv}}$ and $A R E_{\mathrm{rec}}$ Solid line, Hybrid numerical method, dotted line Roe finite-volume method

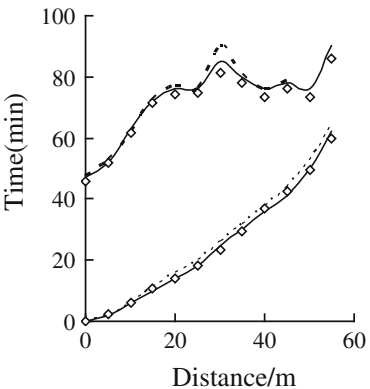

(a) Example 1

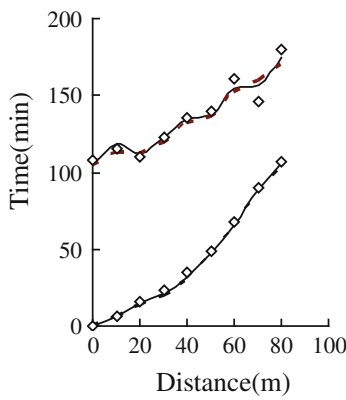

(b) Example 2

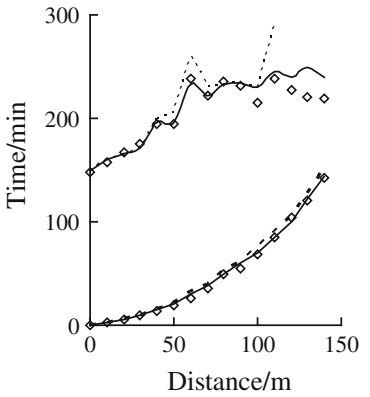

(c) Example 3

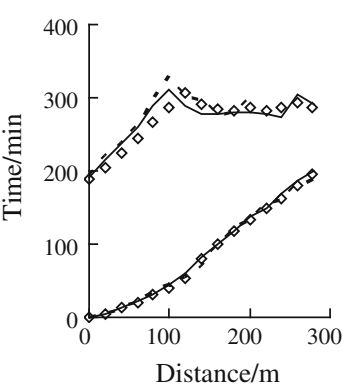

(d) Example 4

— simulated values by hybrid numerical method $\quad$ - - . simulated values by Roe finite volume method $\diamond$ observational values

Fig. 5 Comparison between simulated and observational values by two numerical methods under typical border irrigation examples. Solid line simulated values by hybrid numerical method, dotted line simulated values by Roe finite-volume method, Diamond observational values

of $\Delta h, A R E_{k_{t}, k_{t}+1}, A R E_{\mathrm{adv}}$, and $A R E_{\mathrm{rec}}$, computational efficiency between both numerical methods was compared and analyzed under the same measurement circumstances of numerical stability, convergence, and computational precision. The results in Table 3 show that computational efficiency of the hybrid numerical method is about two times higher than it is in the Roe finite-volume method. Therefore, the proposed model can effectively improve computational efficiency and increase the practicality of border irrigation numerical simulations.

Table 2 Average relative errors between simulated and observational values by two numerical methods under typical border irrigation examples

\begin{tabular}{|c|c|c|c|c|c|}
\hline Average relative error & Numerical method & Sample $1(\%)$ & Sample $2(\%)$ & Sample $3(\%)$ & Sample $4(\%)$ \\
\hline \multirow[t]{2}{*}{$A R E_{\text {adv }}$} & Hybrid numerical method & 4.0 & 3.2 & 6.3 & 6.1 \\
\hline & Roe finite-volume method & 5.6 & 4.6 & 8.6 & 8.7 \\
\hline \multirow[t]{2}{*}{$A R E_{\text {rec }}$} & Hybrid numerical method & 8.3 & 7.3 & 6.1 & 7.8 \\
\hline & Roe finite-volume method & 10.1 & 9.0 & 8.3 & 10.4 \\
\hline
\end{tabular}


Table 3 Computational efficiency comparison of the two numerical methods

\begin{tabular}{lll}
\hline $\begin{array}{l}\text { Border irrigation } \\
\text { examples }\end{array}$ & Numerical method & $\begin{array}{l}\text { Calculation } \\
\text { efficiency/min }\end{array}$ \\
\hline 1 & Hybrid numerical method & 0.92 \\
& Roe finite-volume method & 0.29 \\
& Hybrid numerical method & 0.64 \\
3 & Roe finite-volume method & 0.19 \\
& Hybrid numerical method & 0.37 \\
4 & Roe finite-volume method & 0.11 \\
& Hybrid numerical method & 0.16 \\
& Roe finite-volume method & 0.05 \\
\hline
\end{tabular}

\section{Conclusions}

Each vector of a one-dimensional complete hydrodynamic model of border irrigation was discretized and solved by using a hybrid numerical method. The Crank-Nicolson implicit time scheme was implemented to the $\partial \mathbf{U} / \partial t, \partial \mathbf{F} / \partial x$, and $\mathbf{S}_{2}$, and the $\mathbf{S}_{1}$ and $\mathbf{S}_{3}$ were discretized by using an explicit time scheme. Based on the reconstructed value of each variable on both faces of the cell boundary by using the variable value of the cell center to achieve second-order accuracy, the finite-difference method, the finite-volume method, and the finite-element method were, respectively, employed to discretize the space derivative of the physical flux linear approximation from the implicit time scheme, the space derivative of the physical flux, and the bottom element vector. The Thomas method was used to solve the time-space discretized algebraic equation group with coefficient matrix of a three-diagonal matrix shape, and the one-dimensional complete hydrodynamic model of border irrigation based on the hybrid method was established. By comparing these results with those obtained by using the Roe finite-volume method, the hybrid numerical method presents better numerical stability and convergence with little water quantity-balance error and a low average relative error. The computational efficiency was about two times higher under the same measurement circumstance. The one-dimensional complete hydrodynamic model developed in this study was a better numerical simulation tool in terms of practicality and can improve numerical stability and convergence as well as increase computational precision and efficiency.

Acknowledgments This research was supported by the Projects of the National High-tech R\&D Program No. 2006AA100210 and 2006AA100211.

\section{References}

Bradford SF, Katopodes BF (2001) Finite volume model for non-level basin irrigation. J Irrig Drain E ASCE 127(4):216-223

Bradford SF, Sanders BF (2005) Performance of high-tesolution, nonlevel bed, shallow-water models. J Eng Mech ASCE 131(10):1073-1081

Brufau P, García-Navarro P, Playán E, Zapata N (2000) Numerical modeling of basin irrigation with an upwind scheme. J Irrig Drain E ASCE 128(4):212-223

Chen CL (1970) Surface irrigation using kinematic-wave method. J Irrig Drain D ASCE 96(1):39-46

García-Navarro P, Playán E, Zapata N (2000) Solute transport modeling in overland flow applied to fertigation. J Irrig Drain E ASCE 126(1):33-40

Hildebrand FB (1974) Introduction to numerical analysis. McGrawHill, New York

Lei ZD, Yang SX, Xie SC (1988) Soil water dynamics. Tsinghua University Press, Beijing (in Chinese)

LeVeque RJ (2002) Finite volume methods for hyperbolic problems. The press syndicate of the University of Cambridge, Cambridge

Li YN (1999) Basin irrigation in north China: evaluation, modeling and design for improvement. ISA, Lisbon

Liou MS (1996) A sequel to AUSM: AUSM $^{+}$. J Comput Phys 129(19):364-382

Pieg L (1989) Modifying the shape of rational B-splines, part 1: curves. CAD 21(9):509-518

Strelkoff TS, Katopodes ND (1977) Border irrigation hydraulics with zero-inertia. J Irrig Drain D ASCE 103(3):325-342

Strelkoff TS, Tamimi AH, Clemmens AJ (2003) Two-dimensional basin flow with irregular bottom configuration. J Irrig Drain E ASCE 129(6):391-401

Vivekanand S, Bhallamudi MS (1996) Hydrodynamic modeling of basin irrigation. J Irrig Drain E ASCE 123(6):407-414

Walker WR, Skogerboe GV (1987) Surface irrigation. Theory and practice, Prentice-Hall

Xu D, Cai LG, Wang SL (2000) Farmland water and soil management of agriculture sustainable development. China Water Power Press, Beijing (in Chinese)

Xu D, Cai LG, Mao Z (2004) Water-saving decision technology research in Yellow River irrigation districts. China Agricultural Press, Beijing (in Chinese) 